\title{
Induction of Labour with Isosorbide Mononitrate Versus Prostaglandin E2 (PGE2) in Primigravidas (PGs) at Term
}

\section{Rubina Waheed, ${ }^{1}$ Nadia Ashraf, ${ }^{2}$ Nadia Sabeen, ${ }^{3}$ M Ikram U Allah, ${ }^{4}$ Qaiser Javed,${ }^{5}$ Ayesha khalid, ${ }^{6}$}

\begin{abstract}
Objective: To compare the frequency of caesarean section due to fetal distress with Isosorbide mononitrate versus PGE2 for the induction of labour in primigravida at term.

Methods: It was Randomized Control Trial conducted in Unit I, Department of Obstetrics \& Gynaecology, Lahore General Hospital, Lahore from 11th April 2015 to 10th October 2015. 230 primigravida were randomized into two groups i.e. Prostaglandin E2 and Isosorbide mononitrate for induction of labour. The difference in the frequency of caesarean section due to fetal distress with Prostaglandin E2 and Isosorbide mononitrate for induction of labour in the primigravidas at term was calculated as outcome.

Results: The mean age of $27.62 \pm 3.909$ ranging from 21 to 34 years. The frequency of cesarean section was $12.2 \%$ in Prostaglandin E2 group while $0 \%$ in Isosorbide mononitrate. There was no effect of gestational age, age of mother and number of doses on outcome.

Conclusion: There is no difference in frequency of cesarean section in primigravida groups induced by either Isosorbide mononitrate versus prostaglandin E2. Wherever applicable and feasible we should opt for Isosorbide mononitrate for induction of labour.

Keywords: Cesarean section, Prostaglandin E2, Isosorbide mononitrate, Dinoprostone, Induction of labour, Primigravida

How to cite: : Waheed R., AshrafN., Sabeen N., U Allah I. M., Javed Q., Khalid A. Induction of Labour with Isosorbide Mononitrate Versus Prostaglandin E2 (PGE2) in Primigravidas (PGs) at term. Esculapio 2021;17(02):167-170.

DOI: https://doi.org/10.51273/esc21.2517211
\end{abstract}

\section{Introduction}

Induction of labor is widely carried out all over the world for several fetal and maternal indications. ${ }^{1}$ In the United States induction of labour continues to rise, with $22.5 \%$ of all the births in 2006 reported after induction which is twofold increase since $1990 .^{2}$ Induction of labour in unfavorable cervix often results in prolonged labor or failed induction with increased risk of operative delivery and morbidity. ${ }^{1}$ About $20 \%$ of

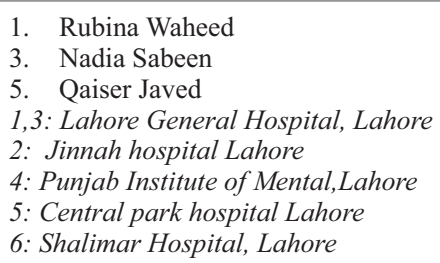

Dr. Rubina Waheed, Department of Gynae and Obst. Lahore General Hospital, Lahore. Email:mailto:rubinawaheed13@yahoo.com" rubinawaheed13@yahoo.com

$\begin{array}{ll}\text { Submission Date: } & 31-03-2021 \\ \text { 1st Revision Date: } & 13-04-2021 \\ \text { Acceptance Date: } & 17-05-2021\end{array}$

pregnant women undergo induction of labour in the UK. In primigravidas, approximately induction to delivery time is 27 hours and 18 hours required in the cervical ripening phase before labour itself starts. An ideal drug for ripening of cervix would induce adequate cervical ripening without causing uterine contractions. ${ }^{3}$ Induction of labour in the presence of unfavorable cervix is a common indication for the use of prostaglandins. However the use of nitrous oxide donors for cervical ripening and induction of labour increased in last years. ${ }^{4}$ Agarwal et al., reported that the frequency of caesarean section was $40.7 \%$ with PGE2 while $0 \%$ with Isosorbide Mononitrate due to fetal distress. The difference between both groups was significant $(\mathrm{p}<$ 0.001 ) Isosorbide Mononitrate is better in terms of low caesarean sections. ${ }^{5}$ But Mukhtar et al., reported that the frequency of caesarean section due to fetal distress was $6.7 \%$ with PGE2 which was high as compared to Isosorbide mononitrate i.e. 0\%. Although the difference between both groups was insignificant $(\mathrm{p}>$ $0.05){ }^{6}$ 
The rationale of our study is to compare the frequency of caesarean section due to fetal distress with Isosorbide mononitrate versus PGE2 for induction of labor in primigravida at term. In literature mentioned above, ambiguity was observed in results. It has been observed that Isosorbide Mononitrate cause low frequency of caesarean section as compared to PGE2 and Isosorbide Mononitrate is cheap and safer drug but PGE2 is still in practice due to controversy in reported results as quoted above. Through this study we want to confirm that whether Isosorbide Mononitrate is safer than PGE2, so that in future we can implement the use of Isosorbide Mononitrate for induction of labour, thus the proven efficacy of this drug through this study can make it choice of both medical professionals and for patients as well.

\section{Operational definition}

C-Section due to Fetal distressis defined as cesarean section performed because of presumed fetal compromise in the form of abnormal/pathological cardiotocography or the presence of any grade of meconium in the amniotic fluid during first stage of labour.

\section{Methods}

It was randomized contolled trial conducted in the Department of Obstetrics \&Gynecology, Lahore General Hospital from 11th April 2015 to 10th October 2015. 230 cases selected by Non-probability consecutive sampling, 115 cases in each group is calculated with $80 \%$ power of test, $5 \%$ level of significance and taking expected percentage of fetal distress i.e. $0 \%$ with Isosorbide Mononitrate while 6.7\% with PGE2 for induction of labour in PGs at term. Patients included were primigravida of age 18-35 years, females with singleton, cephalic presentation of fetus on ultrasound, gestational age $\geq 37$ weeks according to LMP or early scan with reassuring CTG, ruptured membrane (clear liquor) for $\geq 6$ hours. Patients excluded with cervical dilatation more than 3 or Bishop more than 5. Females with medical disorders like impaired renal or hepatic function, cardiac diseases (abnormal ECG and medical record), known hypersensitivity to prostaglandins or Isosorbide mononitrate. History of severe asthma, hypotension $(\mathrm{BP}<110 / 70 \mathrm{mmHg}$, palpitation and migraine, Cephalopelvic disproportion (assessed on USG).

After the approval from hospital ethical committee, 230 patients fulfilling the selection criteria were included in this study, an informed consent was taken.
Demographic profile (name, age, gestational age (G.A), contact) was obtained. Randomly patients were divided in two groups by using random number table. In group Isosorbide Mononitrate, 40mg of Isosorbide mononitrate was administered in the posterior vaginal fornix and dose was repeated every four hours up to 2 dose. In group PGE2, patients were given $3 \mathrm{mg}$ vaginaltablet containing dinoprostone and dose was repeated with interval of 6 hours upto 2 doses. The Bishop score was reviewed regularly at 6,12 and 24 hours after medication. Uterine contractions and fetal heart rate was monitored intermittently and partogram was maintained. If there were signs of fetal distress, then LSCS was done and caesarean section due to fetal distress was labeled (as per operational definition). All the information was recorded on proforma (attached).

\section{Data Analysis}

The data was entered and analyzed through SPSS version 17. The quantitative variables like age and gestational age was presented as mean \& standard deviation. The frequency and percentage was calculated for caesarean section due to fetal distress. Chi-square test was used to compare caesarean section due to fetal distress in both groups. P-value $<0.05$ was considered as significant. Data was stratified for age and number of doses of either drug. Chi-Square test was used post stratification.

\section{Results}

230 patients were included with mean age of $27.62 \pm$ 3.909 ranging from 21 to 34 years. (Table-I) 156 patients $(67.8 \%)$ in our study population were less than 30 years of age whereas 74 patients $(32.2 \%)$ were either 30 years or more, Gestational age in 139 patients $(60.4 \%)$ were between 37 to 38 weeks while in rest of 91 patients (39.6\%) it was above 38 weeks. Among whole cohort, 14 patients $(6.1 \%)$ of sampled population underwent cesarean section. (Table-II) 90 patients (39.1\%) were administered single dose of drug while in 140 patients $(60.9 \%)$ it was double.

When we cross tabulated both treatment group with outcome (frequency of cesarean section), results came up significant on applying chi square test. Out of 115 patients of PGE2 group, 14 underwent C-section while in Isosorbide not a single patient had C-section. (TableIII)

When we stratified our data of dose of drug in treatment group (PGE2 \& Isosorbide) and cross tabulated 
Table 1: Age Distribution of Sampled Population in Years

\begin{tabular}{cccccc}
\hline & N & Minimum & Maximum & Mean & Std. Deviation \\
\hline Age & 230 & 21 & 34 & 27.62 & 3.909 \\
\hline
\end{tabular}

Table 2: Frequency Distribution of Sample Population by Cesarean Section

\begin{tabular}{cccc}
\hline & & Frequency & Percent \\
\hline Valid & Yes & 14 & 6.1 \\
& No & 216 & 93.9 \\
& Total & 230 & 100.0 \\
\hline
\end{tabular}

Table 3: Cross tabulation between Group \& Cesarean Section

\begin{tabular}{lcccc}
\hline & & \multicolumn{2}{c}{ Cesarean Section } & \multirow{2}{*}{ Total } \\
\cline { 3 - 3 } Group & PGE2 & 14 & Nos & \\
& & $12.2 \%$ & $87.8 \%$ & 115 \\
& Isosorbide & 0 & 115 & $100.0 \%$ \\
& & $0.0 \%$ & $100.0 \%$ & 115 \\
Total & 14 & 216 & $100.0 \%$ \\
& & $6.1 \%$ & $93.9 \%$ & $100.0 \%$ \\
\multirow{2}{*}{ Using Pearson Chi-Square, p value $=\mathbf{0 . 0 0 1}$ (Significant) } \\
\hline
\end{tabular}

with cesarean section, we found, patients with single dose had 3 patients with cesarean section in PGE2 group while in Double dose, PGE2 group had 11 patients with cesarean section. (Table-III)

\section{Discussion}

Labor induction in unfavorable cervix often results in failed induction or prolonged labor with increased risk of operative delivery and morbidity especially in primigravidas. ${ }^{1}$ Delivery before the onset of labour is indicated when the maternal and fetal risks associated with continuing the pregnancy are thought to be higher than induction of labour. Prostaglandin E2 and Isosorbide mononitrate play an important role in cervical ripening (a mechanism of remodelling in which collagen is degraded leading to softening of the cervix) and labour induction.

In our study, among whole cohort, 14 patients $(6.1 \%)$ of sampled population underwent cesarean section. frequency of cesarean section was $12.2 \%$ in Prostaglandin E2 group (all 14 patients in this group) while frequency of cesarean section in Isosorbide mononitrate was $0 \%$. The difference was more significant when we applied chi square test ( $p$ value $<0.01$ ). It implies that although efficacy of Prostaglandin E2 is known regarding induction of labour but frequency of cesarean section remains high secondary to fetal distress.

Our results are comparable with previous studies. In a previous study, Mukhtar et al., reported that the frequency of caesarean section due to fetal distress was $6.7 \%$ with PGE2 which was high as compared to Isosorbide mononitrate i.e. $0 \%$. Although the difference between both groups was insignificant $(p>0.05){ }^{6}$

While in another trial, Agarwal et al., assessed that PGE2 group had higher incidence of caesarean sections (27\% versus $17 \%)$ and uterine tachysystole (15\%) and N-RFH (11\%) compared to $0 \%$ in IMN group. ${ }^{5}$

In our study PGE2 had $12.2 \%$ caesearean rate as compared to nil in isorbide group. In PGE2 6.3\% had single dose and $16.4 \%$ who had caesearean had double dose.

After randomization, we cross tabulated the data for all competing and potential confounding variables like age but there was no significant difference. It depicted that both group had equal distribution with respect to their age.

When we stratified our data of age groups in treatment group (PGE2 \& Isosorbide) and cross tabulated with cesarean section, we found, patients with age below 30 years had 9 patients with cesarean section in Prostaglandin E2 group whereas in above 30 years, Prostaglandin E2 group had 5 patients with cesarean section. It shows that efficacy of Isosorbide mononitrate does not reduce with advancing age.

When we stratified our data of dose of drug in treatment group (PGE2 \& Isosorbide) and cross tabulated with frequency of cesarean section, we found, 3 patients with cesarean section had single dose of Prostaglandin E2 while 11 patient had double dose of Prostaglandin E2. When we stratified our data of gestational age in treatment group (PGE2 \& Isosorbide) and cross tabulated with cesarean section, we found, patients with gestational age from 37 to 38 weeks and above 38 weeks had same number of patients (7) with cesarean section in PGE2 group.

In my study vaginal delivery occurred in $87.8 \%$ of subjects and in $100.0 \%$ of patients in isosorbide nitrate group and it was $93.8 \%$ in single dose group as compared to double dose group in $83.6 \%$ of subjects.

In another study by Dhuan et al showed that 38 patients in group I(nitrate) and 31 in group II(PGE2) had normal vaginal delivery, 7 women of group I (Nitrate) and 12 of group II (PGE2) underwent cesarean section while four women $(8 \%)$ of each group had instrumental delivery and concluded that use of prostaglandins for ripening of cervix and induction of labour causes cervical resistance by reorganizing the collagen fibrils in 
the cervix. It decreases the concentration of sulfated glycosaminoglycan (GAG) which causes reduction in electrostatic interactions and weakening of the interfibrillary network of the cervix. In addition to this, prostaglandins also initiate the myometrium contraction and increase the frequency of caesearean section. ${ }^{8}$

Compared with other prostaglandins, one small trial showed that need of oxytocin was reduced with oral misoprostol. Two trials were done on comparison with oral and vaginal misoprostol using different doses but no significant differences were evident.'

In our study we stratified caesearan rate for age groups in treatment group (PGE2 \& Isosorbide) 5.8\% patients with cesarean section in PGE2 group whereas in above 30 years, PGE2 group had $17.2 \%$ patient with cesarean section. Also gestational age in treatment group (PGE2 $\&$ Isosorbide) and cross tabulated with cesarean section, we found, patients with gestational age from 37 to 38 weeks and above 38 weeks had same number of patients (7) with cesarean section in PGE2 group.

The rate of caesarean section was found to be higher in the PGE2 group than IMN (27\% versus $17 \%)$. In the study by Sharma et all in 2000 reported higher caesarean section rate in PGE2 group compared to IMN and GTN group (60\% versus $40 \%$ ) conforming my study results. ${ }^{10,11}$

\section{Conclusion}

It is concluded that at current sample size we reject the null hypothesis that there is no difference in frequency of cesarean section in primigravida groups induced by either Isosorbide mononitrate versus prostaglandin E2 and accept the alternate hypothesis that Isosorbide mononitrate is a better option for induction of labour as far as frequency of cesarean section due to fetal distress is concerned. In our study, frequency of cesarean section came out $12.2 \%$ in Prostaglandin E2 group while $0 \%$ in Isosorbide mononitrate group. Wherever applicable and feasible we should opt for Isosorbide mononitrate for induction of labour. Further studies should be encouraged in this regard.

\section{Conflict of Interest: None}

\section{References}

1. Morad AWA. Vaginal Isosorbide mononitrate versus transcervical Foley's catheter for cervical ripening and induction of labor. Ain Shams Med J. 2011; 62(10): 993-1002.

2. Martin JA, Hamilton BE, Sutton PD, Ventura SJ, Menacker F, Kirmeyer S, et al. National Vital Statistics Reports. Births: final data for 2006. 2009:57(7) [cited 2014];Available from: www.cdc.gov/nchs/data/ nvsr/ nvsr57/nvsr57_07.pdf.

3. Bollapragada SS, MacKenzie F, Norrie JD, Eddama $\mathrm{O}$, Petrou S, Reid M, et al. Randomised placebo-controlled trial of outpatient (at home) cervical ripening with Isosorbide mononitrate (IMN) prior to induction of labour - clinical trial with analyses of efficacy and acceptability. The IMOP Study. BJOG: Int J Obstet Gynaecol. 2009;116(9):1185-95.

4. Abdellah MS, Hussien M, AboAlhassan A. Intravaginal administration of Isosorbide mononitrate and misoprostol for cervical ripening and induction of labour: a randomized controlled trial. Arch Gynecol Obstet. 2011;284(1):25-30.

5. Agarwal K, Batra A, Batra A, Aggarwal A. Randomized Comparison of Isosorbide Mononitrate and PGE2 Gel for Cervical Ripening at Term including High Risk Pregnancy. Int J Reprod Med. 2014;147274:0-5.

6. Mukhtar A, Kazmi F, Rehana F. Comparison of Vaginal Isosorbide Mononitrate with Prostaglandin E2 for Pre-Induction Cervical Ripening at Term. J Rawal Med Coll. 2013;17(1):110-2.

7. Karim SM, Hillier K. Prostaglandins in the control of animal and human reproduction. Br Med Bull 1979; 35:173-7.

8. Duhan N, Hooda M, Sen J. Intracervical dinoprostone versus vaginal isosorbide dinitrate for cervical ripening. 2012;2 (2):269-275

9. Hofmeyr GJ, GülmezogluAM, Alfirevic Z. Misoprostol for induction of labour: a systematic review. Br JObstet Gynaecol. $1999 ; 106(8): 798-803$.

10. Chanrachakul B, Herabutya Y, Punyavachira P. Randomized trial of isosorbide mononitrate versus misoprostol for cervical ripening at term. International Journal of Gynecology and Obstetrics. 2002;78(2):139-145. [PubMed]

11. Sharma Y, Kumar S, Mittal S, Misra R, Dadhwal V. Evaluation of glyceryl trinitrate, misoprostol, and prostaglandin $\mathrm{E} 2$ gel for preinduction cervical ripening in term pregnancy. Journal of Obstetrics and Gynaecology Research. 2005;31(3):210-215

\section{Authors Contribution}

R.W: Conceptualization of Project, Writing of Manuscript

N.A: Data Collection

N.S: Literature Search

M.I.U.A: Statistical Analysis

Q.J: Drafting, Revision

A.K: Data Collection 